Europhys. Lett., 28 (5), pp. 341-346 (1994)

\title{
Optical Evidence of Anderson-Mott Localization in FeSi.
}

L. Degiorgi $\left({ }^{*}\right)$, M. B. Hunt $(*)$, H. R. OTT $\left({ }^{*}\right)$, M. Dressel $(* *)$

B. J. Feenstra $\left({ }^{* *}\right)\left({ }^{\S}\right)$, G. Grüner $\left({ }^{* *}\right)$, Z. Fisk $\left({ }^{* * *}\right)$ and P. CANFIEld $\left({ }^{* * *}\right)$

${ }^{*}{ }^{*}$ Laboratorium für Festkörperphysik ETH-Zürich

CH-809s Zürich, Switzerland

${ }^{(* *)}$ Department of Physics and Solid State Science Center

University of California at Los Angeles - Los Angeles, CA 90024, USA

${ }^{(* * *)}$ Materials Science and Technology Division

Los Alamos National Laboratory - Los Alamos, NM 87545, USA

(received 23 August 1994; accepted 29 September 1994)

PACS. 71.30 - Metal-insulator transitions.

PACS. 78.20 - Optical properties of bulk materials.

\begin{abstract}
We have investigated the electrodynamic response of single-crystalline $\mathrm{FeSi}$ over a frequency range from d.c. to $10^{5} \mathrm{~cm}^{-1}$. At low frequencies, we found evidence for an Anderson-Mott localization behaviour of the low-temperature optical conductivity, while at high frequencies the excitation spectrum resembles that of a conventional semiconductor. The missing spectral weight below the gap energy is redistributed around the gap edge, in disagreement with previous claims based on optical measurements.
\end{abstract}

Among the highly correlated electron systems, various rare-earth compounds known as hybridization-gap semiconductors or Kondo insulators [1] have attracted some interest recently. Because of certain similarities in physical properties, the cubic compound $\mathrm{FeSi}$ was claimed to belong to this class of materials. Of course, this possibility is of high current interest because it would make this material particularly suitable for investigating aspects of the electronic properties of a $d$-transition metal system that might be related with features of correlation effects in $f$-electron materials.

Nearly 25 years ago $\mathrm{FeSi}$ was recognized as a material with puzzling physical properties [2], but only recently has a comprehensive series of low-temperature thermal, magnetic and transport measurements, all made on the same high-quality single crystal, been presented [3]. All these experiments, besides ruling out any type of magnetic ordering at least down to $40 \mathrm{mK}$, can be consistently accounted for by invoking a model of Anderson-localized states associated with residual impurities at the level of $10^{19} \mathrm{~cm}^{-3}$. The latter are most likely due to imperfections in the exact stoichiometry because $\mathrm{FeSi}$ is not a line compound. Furthermore, the electrical conductivity, which is nowhere of activated type below room temperature, appears to saturate below about $5 \mathrm{~K}$, suggesting a "metallic" ground state [3]. Thus, itinerant and Anderson-localized states would have to coexist at low temperatures so that the Fermi level would have to lie at or very close to the mobility edge, the energy which separates the localized from the extended states [4].

(\$) Present address: Solid State Physics Laboratory, Material Science Center, University of Groningen, Nijenborgh 4, 9747 AG Groningen, The Netherlands. 
Consequently, this compound offers the opportunity to study simultaneously the excitation spectrum associated with both types of states. For this purpose, an optical investigation in a very broad frequency range offers the most powerful experimental tool. Previous optical work in the far-infrared and mid-infrared frequency range suggested that FeSi is a dirty metal at high temperatures, while at low temperatures its optical conductivity resembles that of a semiconductor [5]. The most striking and puzzling feature reported in ref. [5] is a redistribution of the missing spectral weight below the gap energy over a very broad frequency range, even extending to several electron volts beyond the covered photon energy range.

We were thus motivated to systematically study FeSi, by investigating a much broader spectral range in energy from the far-infrared (FIR) up to the ultra-violet (UV), and by extending the direct measurement of the optical conductivity to selected millimetre- and micro-wave frequencies. This study was performed on the same sample as was used for the transport, magnetic and thermal investigations [3]. We find that the optical conductivity at low frequencies and low temperatures suggests nearly localized states. At frequencies from FIR up to the mid-infrared (MIR), the excitation spectrum is characterized by several absorptions due to phonon modes but also to mid-gap, or excitonic-like states. Moreover, we found that the spectral weight lost below the "semiconducting" gap, which develops at about $770 \mathrm{~cm}^{-1}$ with decreasing temperature, does reappear in a limited frequency range above it, in contrast to what is claimed in ref. [5].

The reflectivity $R(\omega)$ measurements on our sample, a single crystal grown in antimony flux and having the shape of a prism [3], are summarized in fig. 1a). They were performed over a very broad frequency range from 15 up to $10^{5} \mathrm{~cm}^{-1}$, and as a function of temperature, using four spectrometers with overlapping frequency ranges [6]. The optical conductivity, shown in fig. 1b), is obtained from Kramers-Kronig transformations applied to the measured optical reflectivity. Appropriate extrapolations were used above our highest-frequency limit, while from the FIR down to zero frequency the reflectivity was extrapolated with the Hagen-Rubens law between 300 and $100 \mathrm{~K}$ and to a constant value for the spectra monitored below $40 \mathrm{~K}[6]$.

The most striking feature is the strong temperature dependence of $R(\omega)$ in FIR, where the reflectivity changes from a metallic behaviour at temperatures above $100 \mathrm{~K}$, tending towards $100 \%$ for $\omega \rightarrow 0$, to that of an insulator below $40 \mathrm{~K}$. This can also easily be recognized on plots of the optical conductivity $\sigma_{1}(\omega)$. As the temperature decreases, its low-frequency

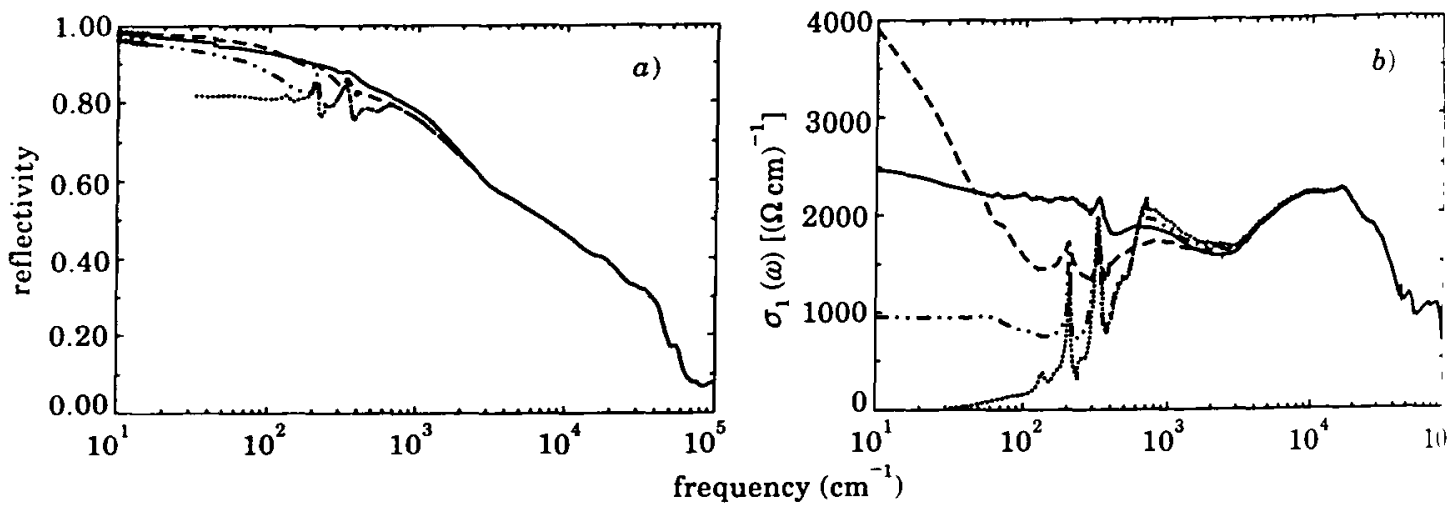

Fig. 1. - a) Optical reflectivity of FeSi at $10(\cdots \cdots), 120(-\cdots-), 200(---)$ and $300 \mathrm{~K}(-), \diamond$ HagenRubens, and $b$ ) corresponding optical conductivity, obtained with Kramers-Kronig transformation (note the logarithmic energy axis). We note that our optical data below $40 \mathrm{~K}$ are all identical to the reported spectrum at $10 \mathrm{~K}$. 


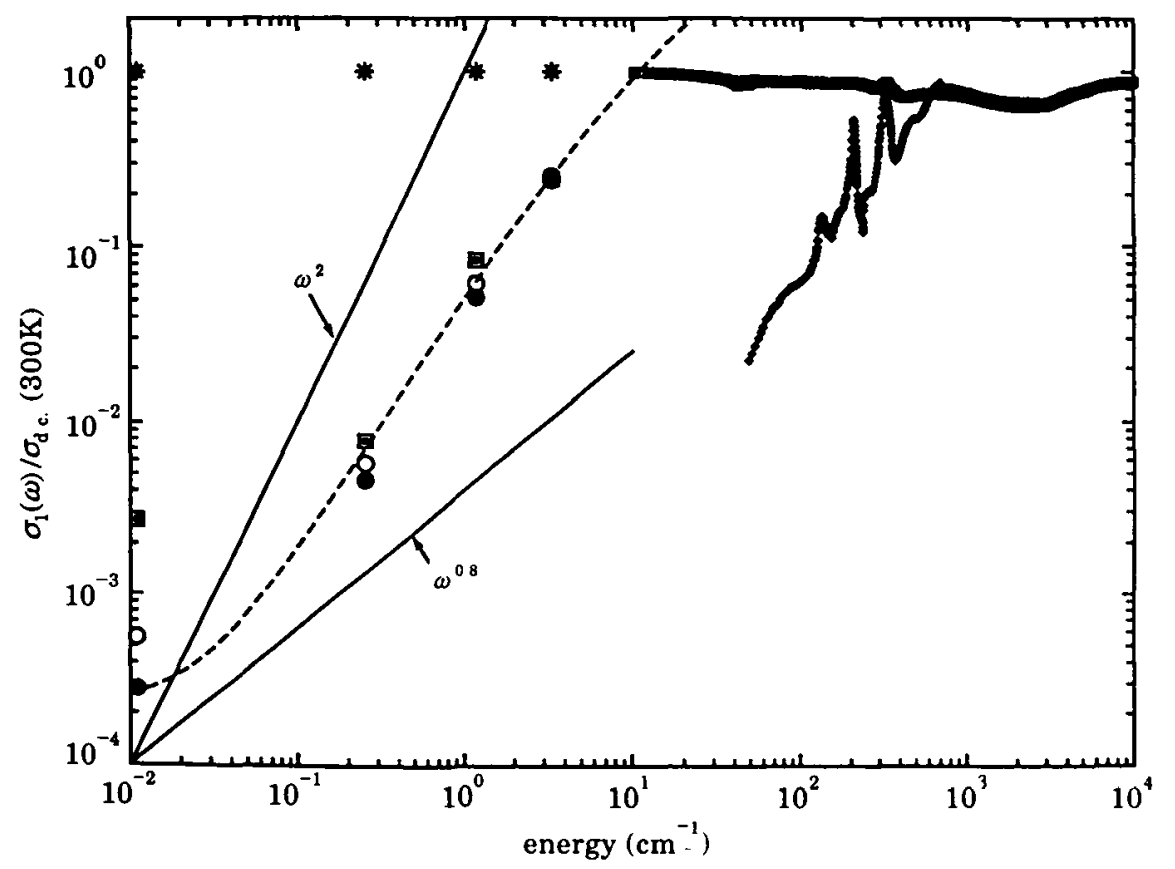

Fig. 2. - Log-log plot of the normalized optical conductivity at $7.4,35$ and $100 \mathrm{GHz}$ at various temperatures $* 300 \mathrm{~K}, \square 10 \mathrm{~K}, \circ 5 \mathrm{~K}, \bullet 1.2 \mathrm{~K}$, together with the FIR-to-visible optical data at $300(\square)$ and $6 \mathrm{~K}(\diamond)$ (see fig. $1 b)$ ). The points on the abscissa axis (i.e. at $10^{-2} \mathrm{~cm}^{-1}$ ) correspond to the d.c. values. A phenomenological fit with eq. (1) is also presented (dashed line: $\sigma_{\mathrm{d} . \mathrm{c}}=0.5413(\Omega \mathrm{cm})^{-1}$, $A=6.6 \cdot 10^{-2} \Omega / \mathrm{cm}$ and $I_{0}=750 \mathrm{~cm}^{-1}$ ).

limit drops continuously, with the exception of the measurements around $200 \mathrm{~K}$, where the d.c. limit is approached only at still lower frequency [7]. Below $40 \mathrm{~K}$ it is vanishing small. As can be seen in fig. $1 b)$, the Drude component in $\sigma_{1}(\omega)$ ascribed to itinerant charge carriers is progressively quenched down to $100 \mathrm{~K}$ and disappears below $40 \mathrm{~K}$. Even though $\hat{f}(T)$ still increases by orders of magnitude below $40 \mathrm{~K}$, we are not able to detect any temperature dependence of $\sigma_{1}(\omega)$ in the FIR range, this part of the spectrum being characterized by an optical conductivity typical of insulators. The increase of $\sigma_{1}(\omega \rightarrow 0)$ at $200 \mathrm{~K}$ is related to a corresponding shallow minimum in $f(T)$ [3]. We also note the appearance of several distinct and narrow modes in the FIR frequency range upon decreasing the temperature to $10 \mathrm{~K}$, in agreement with previous work [5].

In order to gain some additional information on the excitation spectrum of $\mathrm{FeSi}$ at low frequencies, we measured the optical conductivity at $7.4,35$ and $100 \mathrm{GHz}$ by placing the sample or a small piece of it in the maximum of the electric field of a cylindrical $T E_{001}$ microwave cavity. The conductivity was evaluated from the change of the quality factor, using standard cavity perturbation theory [8]. Figure 2 displays in a log-log plot the frequency-dependent optical conductivity for millimetre- and micro-wave frequencies at several temperatures together with the 300 and $10 \mathrm{~K}$ optical results from FIR up to the visible frequency range (see also fig. $1 b$ )). All the data were normalized to the d.c. value at $300 \mathrm{~K}$. The points on the abscissa axis correspond to the d.c. values [3].

As pointed out in the introduction, there is evidence for Anderson-localized states in $\mathrm{FeSi}$ at low frequencies [3]. For such states the optical conductivity $\tau_{1}(\omega)$ at $T=0 \mathrm{~K}$ is expected to approach zero as $\omega^{2}\left[\ln \left(I_{0} / \omega\right)\right]^{4}[4] . I_{0}$ is a characteristic energy related to the overlap 
integral (I) through the expression $I=I_{0} \exp [-\alpha R]$, where $R$ is the distance between localized states and $\alpha$ the rate at which the wave function on the impurity falls off [4]. At non-zero temperature, optically induced hopping is supplemented by phonon-assisted thermal hopping and $\sigma_{1}(\omega)$ follows the formula $\omega k_{\mathrm{B}} T[\ln (\widetilde{\omega} / \omega)]^{4}$. This corresponding frequency dependence is approximated by $\omega^{0.8}$ for $\omega \ll \widetilde{\omega}$, where $\widetilde{\omega}$ is an average phonon frequency [4]. At high frequencies (i.e. $\hbar \omega \gg k_{\mathrm{B}} T$ ) the $\omega^{2}$-dependence will be the leading term in $\sigma_{1}(\omega)$, while at moderate temperatures, i.e. $\hbar \omega<k_{\mathrm{B}} T$, the frequency dependence of $\sigma_{1}$ will approximately follow the $\omega^{0.8}$ behaviour for $\omega \ll \bar{\omega}[4]$. In fig. 2 , we indicate these characteristic limiting behaviours and at high frequencies or low temperatures the data do indeed show the trend to an $\omega^{2}$-dependence. On the other hand, at low frequencies or enhanced temperature the slope of $\sigma_{1}(\omega)$ approaches the $\omega^{0.8}$ variation.

Since the conductivity in the d.c. limit, and at millimetre- and micro-wave frequencies saturates to a constant value below $5 \mathrm{~K}$, we may consider the measurement at the lowest temperature of $1 \mathrm{~K}$ as the $T \rightarrow 0$ limit of $\sigma_{1}(\omega)$. Assuming the dominance of optically activated hopping we may therefore write [4]

$$
\sigma(\omega)=\sigma_{\text {d.c. }}+A \omega^{2}\left[\ln \left(I_{0} / \omega\right)\right]^{4},
$$

where $\sigma_{\text {d.c. }}$ accounts for the finite d.c. conductivity [3] and $A$ is a constant. The dashed line in fig. 2, calculated with the parameters in the figure caption, demonstrates the striking agreement between experiment and the calculation using eq. (1). From fig. 2 it is obvious that for low temperatures the low-frequency $\sigma_{1}(\omega)$ data do not smoothly join the corresponding values obtained in the FIR range. This peculiar behaviour of $\sigma_{1}(\omega)$ is not affected by the extrapolation to zero frequency, used for the Kramers-Kronig transformation, and indicates the existence of a broad maximum in the spectral range around $10 \mathrm{~cm}^{-1}$. A more sophisticated numerical calculation for a photon-induced hopping mechanism by Blinowski and Mycielski [9] shows indeed that the absorption coefficient goes through a maximum in FIR, as has subsequently been confirmed by FIR absorption experiments in $n$-type silicon [10]. Furthermore, several experiments on amorphous glasses (e.g., $\mathrm{As}_{2} \mathrm{Se}_{3}, \mathrm{As}_{2} \mathrm{Te}_{3}$ etc.), although revealing quite substantially different absolute values of $\sigma_{1}(\omega)$, do display a frequency dependence of the optical conductivity which is first linear, then follows a temperature-independent $\omega^{2}$ behaviour and, in the GHz-FIR energy spectral range, either has a broad maximum or saturates to a frequency-independent value [11].

The second relevant feature of the electrodynamic response of FeSi is the MIR absorption at $770 \mathrm{~cm}^{-1}$. It may be associated with a semiconductor-type gap, although $\rho_{\text {d.c. }}$ does not show, in any temperature range, an activated behaviour. This assignment, already suggested by Schlesinger and co-workers [5], is in qualitatively fair agreement with results of band structure calculations [12], even though they predict a larger gap value. From our data (see fig. 1b)) we conclude that the filling of the gap with increasing temperature is not completed up to $300 \mathrm{~K}$ [12]. This is in distinct contrast with the claim of ref. [5], where the disappearance of the gap was monitored at a temperature of about $200 \mathrm{~K}$.

Another important difference with the previously reported optical results [5] concerns the redistribution of the missing spectral weight below the gap. In our data, that spectral weight is essentially recovered at a frequency $\omega_{\mathrm{c}} \sim 4 \omega_{\mathrm{g}}$, where $\omega_{\mathrm{g}}=770 \mathrm{~cm}^{-1}$ is the gap excitation frequency. We have calculated the spectral weight function defined as [5]

$$
\frac{n(\omega)}{m^{*}}=\frac{2}{\pi} \int_{0}^{\omega} \sigma_{1}\left(\omega^{\prime}\right) \mathrm{d} \omega^{\prime},
$$

which is displayed in fig. 3 . It may be seen that the spectral weight which disappeared below the gap is indeed redistributed in a limited frequency range, and that above $3000 \mathrm{~cm}^{-1}$ there 


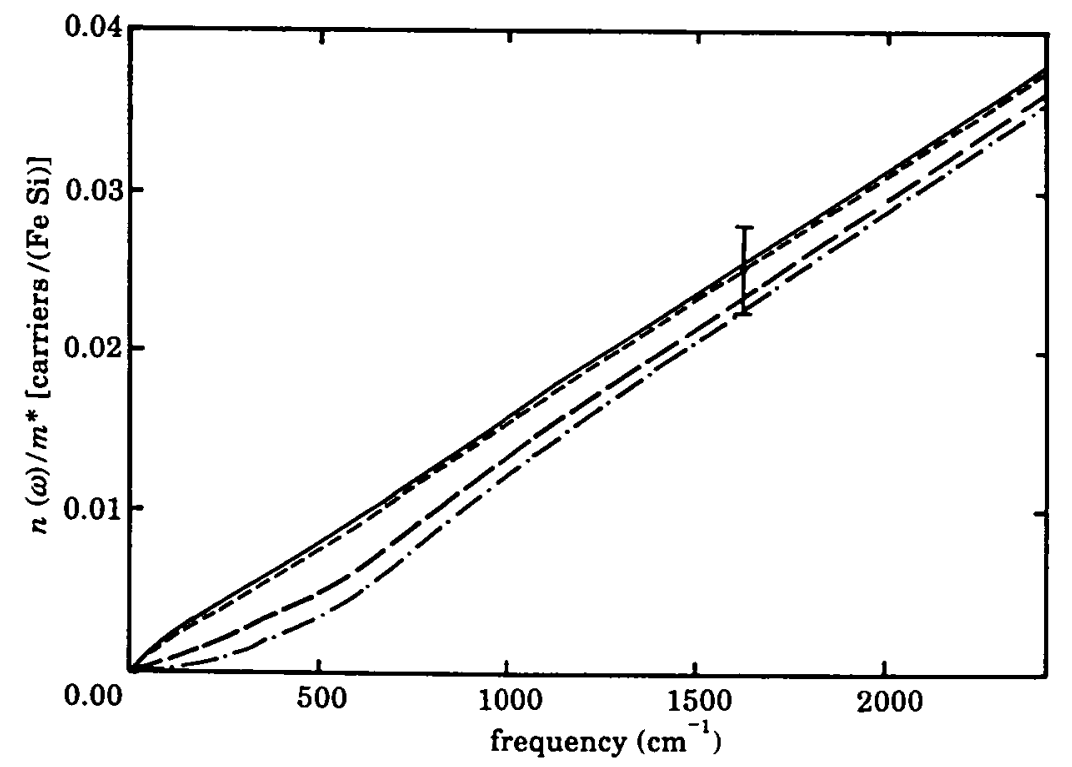

Fig. 3. - Frequency dependence of the spectral-weight sum rule, defined as the ratio between the effective charge carrier concentration up to $\omega$ and the effective mass, at various temperatures: $-.-10 \mathrm{~K},---120 \mathrm{~K}, \ldots-200 \mathrm{~K},-300 \mathrm{~K}$, calculated using eq. (2). The error bar indicates the effect on the spectral weight of shifting the reflectivity by $1 \%$.

is almost no temperature dependence (i.e. all the curves in fig. 3 merge together). This means that there is no need for an integration of $\sigma_{1}(\omega)$ to very high frequencies in order to satisfy the spectral-weight sum rule.

Finally, we briefly discuss the narrow absorption peaks at $140,200,330$ and $460 \mathrm{~cm}^{-1}$. One of them, i.e. at $330 \mathrm{~cm}^{-1}$, persists up to $300 \mathrm{~K}$. We judge the origin of these absorptions to be twofold. An obvious possible source are phonons. The crystal structure of FeSi [12] may be viewed as a distorted $\mathrm{NaCl}$ structure and from group theory considerations one expects three IR-active phonons [13]. The measured elastic constants [14] are indicative of a quite strong ionicity in $\mathrm{FeSi}$, comparable to the situation met in $\mathrm{MgO}$ [5]. This might allow for phonon modes at fairly high frequencies, as high as $300 \mathrm{~cm}^{-1}$. Therefore, while the low-energy absorptions at 140 and $200 \mathrm{~cm}^{-1}$ are almost certainly due to IR-active vibration states, the shoulder at $460 \mathrm{~cm}^{-1}$, overlapping the gap excitation, and most likely also the sharp and narrow one at $330 \mathrm{~cm}^{-1}$, are too high in energy to be assigned to phonon modes.

There is, however, evidence which suggests that these latter absorptions are due to excitations between "mid-gap" localized states and the continuum of the valence and conduction bands. Invoking the rather simple hydrogen model for states in a semiconducting gap, the impurity energy levels are given by $E_{\mathrm{n}}=\left(m^{*} R y\right) /\left(\varepsilon_{\mathrm{eff}}^{2} n^{2}\right), m^{*}$ being the effective mass, $\varepsilon_{\text {eff }}$ the effective dielectric function in the frequency range where excitations are expected [15] and $R y=13.6 \mathrm{eV}$. The effective band mass may be deduced from electronicband-structure calculations [12], while $\varepsilon_{\text {eff }}$ can be calculated with the model of Mahanti and Varma [16], who considered the problem of exciton states in the presence of interactions of electrons with optical phonons. Since the phonon modes are at energies also expected for the excitonic-like absorptions, one cannot set $\varepsilon_{\text {eff }}$ equal to the static dielectric constant $\varepsilon_{0}=$ $=\varepsilon_{1}(\omega \rightarrow 0)$, as is usually done [15]. With $m^{*} \sim 5-10$ [12] and $\varepsilon_{\text {eff }}=60$, the sharp features at 330 and $460 \mathrm{~cm}^{-1}$ would indeed be consistent with absorptions of excitonic type. In addition, 
spectral-weight estimates lead to values for the corresponding mode strengths consistent with an impurity concentration of the order of $10^{20} \mathrm{~cm}^{-3}$, which is in fair agreement with a previous evaluation based on thermal and transport properties [3]. This adds support to our interpretation in terms of localized excitonic states.

In conclusion, by applying various experimental techniques we have determined the complete electrodynamic response of the non-magnetic narrow-gap semiconductor FeSi, spanning from millimetre- and micro-wave frequencies up to the ultra-violet spectral range. At low temperatures, the frequency dependence of $\sigma_{1}(\omega)$ is compatible with Anderson(weakly)-localized states, which confirms the conclusions arrived at by interpreting d.c. transport and thermodynamic experiments [3]. The excitation spectrum, due to extended states (at high frequencies), looks very similar to that of a conventional semiconductor with an energy gap which fills in progressively but not completely up to $300 \mathrm{~K}$. In this respect, the total spectral weight is fully conserved at each temperature and what is lost below the gap is recovered in an extended but limited frequency range above the gap. We see no need for an extreme high-energy scale for explaining the electronic properties of FeSi.

The authors are grateful to R. MonNIER, K. BEDELl and P. WACHTER for helpful discussions, to J. MÜLLER for technical assistance and to the Swiss and US National Foundations for Scientific Research for financial support. One of us (MD) wishes also to thank the Alexander von Humboldt Foundation for financial support. One of us (BJF) wishes to acknowledge the Netherlands Organization for Scientific Research (NWO).

\section{REFERENCES}

[1] Aeppli G. and Fisk Z., Comments Condens. Matter Phys., 16 (1992) 155.

[2] Wolfe R., Wernick J. H. and Haszko S. E., Phys. Lett, 19 (1965) 449; Jaccarino V., Wertheim G. K., Wernick J. H., Walker L. R. and AraJs S., Phys. Rev., 160 (1967) 476.

[3] Hunt M. B., Chernikov M. A., Felder E., OtT H. R., Fisk Z. and Canfield P., to be published in Phys. Rev. B.

[4] Mort N. F. and Davis E. A., Electronic Processes in Non-Crystalline Materials (Clarendon Press, Oxford) 1971, and references therein.

[5] Schlesinger Z., Fisk Z., Zhang H. T., Maple M. B., DiTusa J. F. and Aeppli G., Phys. Rev: Lett., 71 (1993) 1748.

[6] Awasthi A. M., Degiongi L., Grüner G., Dalichaouch Y. and Maple M. B., Phys. Rev. B, 48 (1993) 10692.

[7] Degiorgi L., Ott H. R., Dressel M., Feenstra B. J., Grüner G. and Fisk Z., unpublished.

[8] KLEIN 0. et al., Int. J. Infrared Millimeter Waves, 14 (1993) 2423; Donovan S. et al., Int. J. Infrared Millimeter Waves, 14 (1993) 2459; DRESSEL M. et al., Int. J. Infrared Millimeter Waves, 14 (1993) 2489.

[9] Blinowski J. and Mycielski J., Phys. Rev., 136 (1964) A-136.

[10] Milward R. C. and Neuringer L. J., Phys. Rev. Lett., 15 (1965) 664.

[11] Pollak M., Philos. Mag., 23 (1971) 519, and references therein.

[12] Mattheiss L. F. and Hamann D. R., Phys. Rev. B, 47 (1993) 13114; Fu C., KriJn MP. C. M. and DoniaCH S., Phys. Rev. B, 49 (1994) 2219.

[13] Fateley W. G., Dollish F. R., McDevitT N. J. and Bentley F. F., Infrared and Raman Selection Rules for Molecular and Lattice Vibrations (J. Wiley, New York, N.Y.) 1972.

[14] Zinov'eva G. P., Andreeva L. P., Gel'd P. V. and Krentsis R. P., Sov. Phys. Solid State, 15 (1974) 1469.

[15] Bassani F. and Pastori Parravicini G., Optical Transitions in Solids (Pergamon Press, Oxford) 1975.

[16] Mahanti S. D. and Varma C. M., Phys. Rev. Lett., 25 (1970) 1115. 